\title{
A pre-relaxed FBG accelerometer using transverse forces with high sensitivity and improved resonant frequency
}

\author{
Kuo Li, Yuqing Li,* Guoyong Liu, Juan Tian, and Yanchun Wang \\ Bengbu University, Department of Electronic and Electrical Engineering, 1866 Caoshan Rd, Bengbu, China, 233030
}

Received July 09, 2019; accepted March 27, 2020; published March 31, 2020

\begin{abstract}
Fiber Bragg grating (FBG) accelerometers using transverse forces have higher sensitivity but lower resonant frequency than ones using axial forces. By shortening the distance between the two fixed ends of an FBG, its resonant frequency can be improved without lowering the sensitivity. Here, a compact FBG accelerometer using transverse forces with a slightly pre-relaxed FBG and a $25 \mathrm{~mm}$ distance between the two fixed ends has been demonstrated with the crest-to-trough sensitivity $1.1 \mathrm{~nm} / \mathrm{g}$ at $5 \mathrm{~Hz}$ and the resonant frequency $42 \mathrm{~Hz}$. It reveals that making the FBG slightly pre-relaxed rather than pre-stretched also improves the tradeoff between its sensitivity and resonant frequency.
\end{abstract}

Fiber Bragg grating (FBG) sensors have many intrinsic advantages, such as frequency modulation and ease of multiplexing $[1 \div 2]$. Many different FBG sensors have been explored to monitor acceleration, wind speed, etc $[3 \div 8]$. When an FBG is fixed by its two ends, it is more sensitive to transverse forces than axial ones [9]. Sensitive FBG accelerometers using transverse forces have been developed [10-12]. An inertial object is hung or fixed at the middle of the two fixed ends of the FBG. If the accelerometer is subjected to vibration, the inertial object will vibrate along with it. The part between the two fixed ends of the FBG is called, here, "vibration arm", as it vibrates along with the inertial object. A biaxial FBG accelerometer, with a 1.76 gram inertial object and a 50mm-long vibration arm, used two FBGs to measure transverse and axial forces [11]. The difference between the resonant wavelengths of the two FBGs showed the acceleration in the axial direction, whereas the average showed the acceleration in the transverse direction. In the transverse direction, the crest-to-trough sensitivity $0.55 \mathrm{~nm} / \mathrm{g}$ at $5 \mathrm{~Hz}$ and resonant frequency $34 \mathrm{~Hz}$ were achieved, being 24 and 1/26 times of those in the axial direction, respectively [11]. Therefore, FBG accelerometers using transverse forces have higher sensitivity but lower resonant frequency than ones using axial forces.

Only for FBG accelerometers using transverse forces, the pre-stretch (the tension of the FBG when it is fixed and before a transverse force is applied) influences its sensitivity and resonant frequency. As the pre-stretch increases, the sensitivity always decreases, but the resonant frequency first decreases then increases [12]. The

*E-mail:lyq@bbc.edu.cn crest-to-trough sensitivities 1.24 and $0.77 \mathrm{~nm} / \mathrm{g}$ at $5 \mathrm{~Hz}$ and the resonant frequencies 26.1 and $40.9 \mathrm{~Hz}$ were achieved, by using 5.71 and 3 gram inertial objects, $50 \mathrm{~mm}$-long and $24 \mathrm{~mm}$-long vibration arms, and 0.36 and $0.16 \mathrm{~nm}$ pre-stretched FBGs, respectively [12]. The resonant frequency $26.1 \mathrm{~Hz}$ decreased to $23.9 \mathrm{~Hz}$ as the pre-stretch increased from $0.36 \mathrm{~nm}$ to $1.1 \mathrm{~nm}$ and increased to $31 \mathrm{~Hz}$ as the pre-stretch increased to $3.22 \mathrm{~nm}$.

In this paper, we deal with an FBG accelerometer using transverse forces with a slightly pre-relaxed FBG. When compared, the one described above, with sensitivity $0.77 \mathrm{~nm} / \mathrm{g}$, resonant frequency $40.9 \mathrm{~Hz}$, inertial object 3 gram, vibration arm $24 \mathrm{~mm}$, had a higher sensitivity and similar resonant frequency (sensitivity $1.1 \mathrm{~nm} / \mathrm{g}$, resonant frequency $42 \mathrm{~Hz}$, inertial object 5.17 gram, vibration arm $25 \mathrm{~mm})$. The higher sensitivity was due to the heavier inertial object. The similar resonant frequency was due to the slightly pre-relaxed FBG; otherwise, the resonant frequency would have been lower. The fact that making the FBG slightly pre-relaxed rather than pre-stretched improves the tradeoff has been firstly reported here, as far as we know.

Figure 1 shows the packed FBG accelerometer in comparison with a coin. Its length, width and height were 40, 18 and $16 \mathrm{~mm}$, respectively. The FBG (Da Hui Photoelectric Inc., Jinan, China) was fixed by its two ends by using first epoxy glue and then 4 bolts pressing down 2 metal pieces. An inertial object was hung in the middle of the FBG. The distance between the two fixed ends was $25 \mathrm{~mm}$. The Bragg grating was $10 \mathrm{~mm}$ in length, $3 \mathrm{~dB}$ width $0.23 \mathrm{~nm}$, and free state resonant wavelength $1533.34 \mathrm{~nm}$. The resonant wavelength was $1534.02 \mathrm{~nm}$ at equilibrium after the inertial object was hung. The mass of the inertial object was 5.17 gram. The stretch at equilibrium was $1534.02-1533.34=0.68 \mathrm{~nm}$. By using the results in Ref. [9], 5.17 gram corresponds with $0.507 \mathrm{~N}$ transverse force and $0.82 \mathrm{~nm}$ resonant wavelength change at $0 \mathrm{~nm}$ pre-stretch, assuming the FBGs used had similar mechanical parameters. A non-zero pre-stretch will result in an even larger resonant wavelength change at equilibrium (the sum of the pre-stretch and the resonant wavelength change due to the inertial object). The resonant 
wavelength change at equilibrium of the accelerometer with a 5.71 gram inertial object always increased as the pre-stretch increased from 0.03 to $0.69 \mathrm{~nm}$, from 0.69 to $1.41 \mathrm{~nm}$, from 1.41 to $1.93 \mathrm{~nm}$, from 1.93 to $3.16 \mathrm{~nm}$, respectively [12]. Therefore, here, as the resonant wavelength change at the equilibrium of $0.68 \mathrm{~nm}$ was smaller than that of $0.82 \mathrm{~nm}$ at zero pre-stretch, the FBG was slightly pre-relaxed.

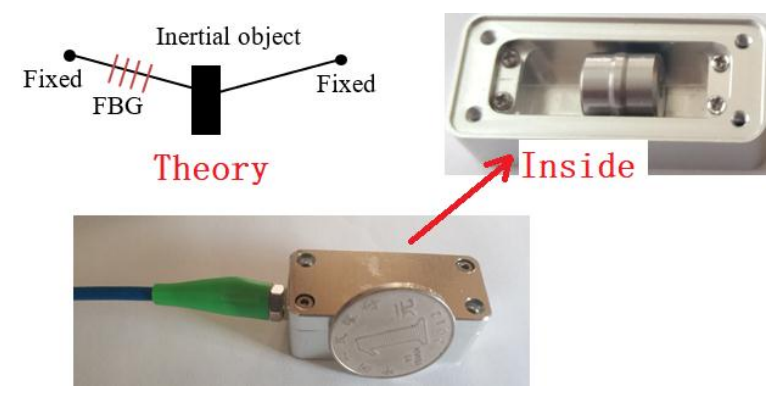

Fig. 1. Packed FBG accelerometer.

Unlike the pre-stretch which can be measured by the resonant wavelength change when the FBG is fixed and before a transverse force is applied, the pre-relax cannot. But after a transverse force is applied, the pre-relax reduces its induced resonant wavelength change. In terms of FBG accelerometers using transverse forces, the pre-relax will reduce the sensitivity. Therefore, a large pre-relax probably is not ideal here.

Figure 2 shows the experimental setup. The FBG accelerometer and a piezo accelerometer (CT1010L, Chengke Inc., Shanghai, China) were placed together on top of a shaker (QSCGQ-ZT1, Zhejiang Qiushi Teaching Apparatuses Inc. Zhejiang, China). The shaker was fed with a sinusoidal wave generated by a signal generator (MFG2160MR, Gwinstek Inc, Taiwan). Both of the FBG and Piezo accelerometers were sampled at $500 \mathrm{~Hz}$. The Piezo accelerometer showed a resolution of $0.001 \mathrm{~g}$; the FBG interrogator (SAI-1127AF, Casstk Inc., Shenzhen, China) shown a resolution of $1 \mathrm{pm}$.

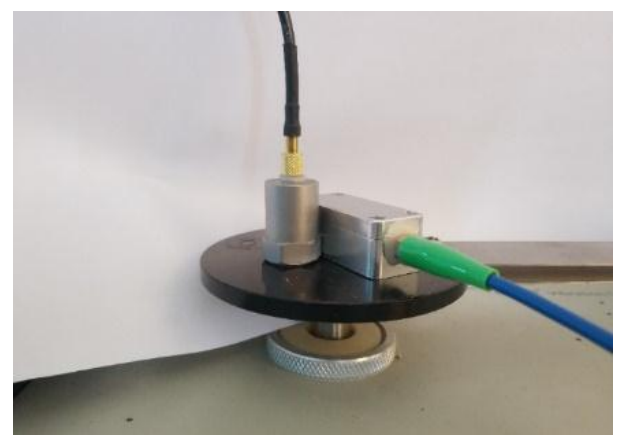

Fig. 2. Experimental setup.
Figure 3 shows the results of the two accelerometers at $5 \mathrm{~Hz}$ at different accelerations and data processing method. The frequency of the input signal of the shaker was fixed at $5 \mathrm{~Hz}$, and 6 different amplitudes were applied. Each amplitude was kept for several seconds, and then increased into next until the last one. The two insets in the first row show the overall results. As both accelerometers were sampled at $500 \mathrm{~Hz}$, one data point was recorded every $2 \mathrm{~ms}$. The time axes refer to the data points. For example, the two insets in the second row show 1 second data extracted from the overall results by using 500 data points each. The two insets in the last row show the FFT results of the 1 second data, respectively.
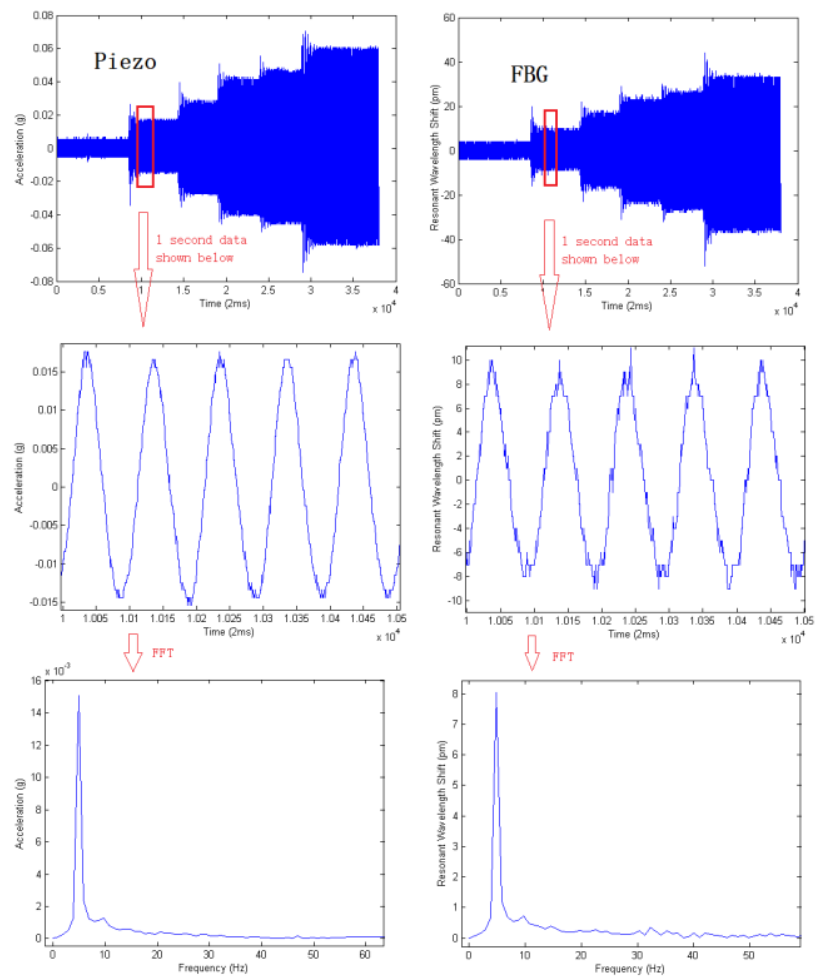

Fig. 3. Experimental results and data processing method (a larger Fig. can be found in the supplementary material).

Figure 4 shows the sensitivity of the FBG accelerometer based on the FFT results of all the 6 accelerations. The sensitivity was the slope of the line, $1.1 \mathrm{~nm} / \mathrm{g}$. The FFT results of the piezo and FBG accelerometers at the 6 accelerations correspond with the horizontal and vertical coordinates of the 6 non-zero points. The FFT results show the amplitudes of the accelerations. The crest-to-trough wavelength change is the twice of the amplitudes (the FFT results of the FBG accelerometer).

Figure 5 shows the frequency response of the FBG accelerometer. It was obtained in a similar way as the sensitivity. However, the frequency of the input signal of the shaker was adjusted here, and the sensitivities (the ratio 
of wavelength change to acceleration) were calculated just for one acceleration, instead of 6 accelerations. It shows that the resonant frequency was $42 \mathrm{~Hz}$.

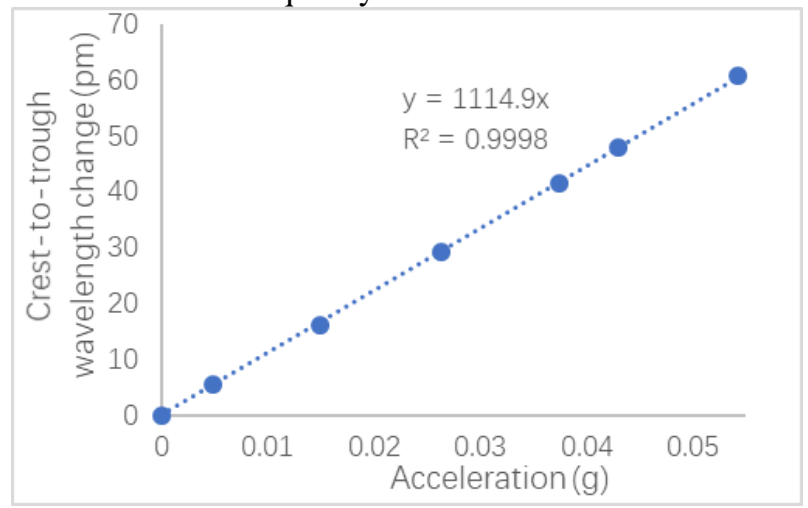

Fig. 4. Crest-to-trough sensitivity at $5 \mathrm{~Hz}$.

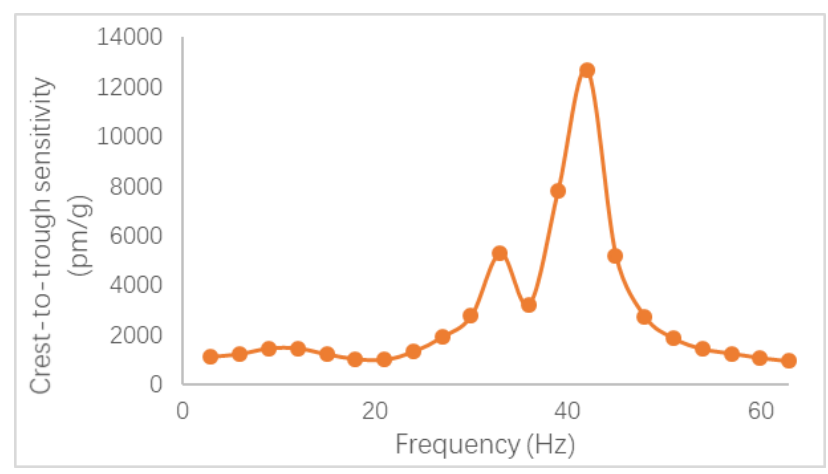

Fig. 5. Frequency response

In conclusion, a compact FBG accelerometer using transverse forces is demonstrated with an excellent tradeoff between the sensitivity and resonant frequency, because of (1) its short distance $25 \mathrm{~mm}$ between the two fixed ends of the FBG and (2) the fact that the FBG was slightly pre-relaxed rather than pre-stretched. While shortening the distance between the two fixed ends of the FBG will always improve the tradeoff, making the FBG slightly pre-relaxed rather than pre-stretched may also help.

This research was funded by Bengbu University, grant number BBXY2018KYQD02, 2019ZR04zd, 2017ZR06 and 2017ZR10, and Anhui Province Education Department KJ2019A0853 and KJ2017A565.

\section{References}

[1] B.S. Kawasaki, K.O. Hill, D.C. Johnson, Y. Fujii, Opt. Lett. 3, 66 (1978).

[2] K.O. Hill, G. Meltz, J. Lightwave Techn. 15, 1263 (1997).

[3] B. Lee, Opt. Fiber Techn. 9, 57 (2003).

[4] A. Laudati, F. Mennella, M. Giordano, G. D'Altrui, C.C. Gassini, A. Cusano, IEEE Phot. Techn. Lett. 19, 1991 (2007).

[5] P.F. Costa Antunes, C.A. Marques, H. Varum, P.S. Andre, IEEE Sens. J. 12, 2399 (2012).

[6] Y. Guo, D. Zhang, Z. Zhou, L. Xiong, X. Deng, Chinese Opt. Lett. 11, 21 (2013).

[7] Y. Zhang, W. Zhang, Y. Zhang, L. Chen, T. Yan, S. Wang et al. , IEEE Sensors J. 17, 614 (2017).

[8] X.-B. Zhu, Optoelectr. Lett. 14, 276 (2018).

[9] K. Li, M.H. Yau, T.H.T. Chan, D. Thambiratnam, H.Y. Tam, Opt. Lett. 38, 311 (2013).

[10] K. Li, T.H.T. Chan, M.H. Yau, T. Nguyen, D.P. Thambiratnam, H.Y. Tam, Appl. Opt. 52, 6401 (2013).

[11] K. Li, T.H.T. Chan, M.H. Yau, D.P. Thambiratnam, H.Y. Tam, IEEE Phot. Technol. Lett. 26, 1549 (2014).

[12] K. Li, T.H. Chan, M.H. Yau, D.P. Thambiratnam, H.Y. Tam, Appl. Opt. 53, 1200 (2014). 